\title{
PAPER
}

\section{Tissue and cell imaging in situ: potential for applications in pathology and endoscopy}

\section{J-Y Scoazec}

Gut 2003;52(Suppl IV):iv1-iv6

Morphological sciences have recently experienced a significant technological breakthrough that offers new opportunities for cell and tissue imaging in situ but also raises new challenges to pathologists, who must adapt to a rapidly evolving environment. New partners, such as cell and molecular biologists, have provided pathologists with highly powerful tools for cell and subcellular imaging. They include: (a) the adaptation of techniques derived from molecular biology and cytogenetics, (b) the development of new microscopic tools, such as confocal microscopy, and (c) the emergence of new preparative techniques, such as microdissection or tissue arrays. However, recent technological progresses in various fields, from endoscopy to genomics, also raise new challenges to pathologists. Pathologists must therefore be prepared to redefine their area of expertise: this will be achieved through a continuous collaboration with all the partners involved in cell and tissue imaging and analysis but also by emphasising the importance of the informations provided by cell and tissue imaging in situ.

Correspondence to: Dr J-Y Scoazec, Service Central d'Anatomie et Cytologie Pathologiques, INSERM U45, Hôpital Edouard Herriot, Lyon, et Plate-forme

d'Histopathologie du Petit Animal (ANIPATH), Génopôle Rhône-Alpes, France;

jean-yves.scoazec@chu-lyon.fr
B iological objects may be imaged by different observers, for different purposes, and with different tools. Several levels of observation, corresponding to increasing degrees of complexity, may be distinguished: (1) at the organ level, whole organs or even whole organisms are explored, (2) at the tissular level, the observer focuses on the general architecture of living tissues, (3) at the cellular level, the observer deals with the identification of cell lineages and with the study of cell structure and functions, (4) at the subcellular level, the cellular architecture and the various compartments of living cells may be explored, and (5) at the molecular level, the numerous molecular constituants of living cells may be identified and characterised.

At one end of the spectrum, the organ and tissue levels are the field of expertise of clinical imagers, using techniques of in vivo imaging, such as radiology and endoscopy. At the other end of the spectrum, the subcellular and molecular levels are the fields of expertise of cell biologists, biochemists, and molecular biologists. Between these extremes, a very large field of investigation, including the tissular, cellular, and subcellular levels, has been historically devoted to the morphological sciences, including histology, em- bryology, and pathology. Morphologists have developed techniques of ex vivo imaging, which can be applied only to biological specimens submitted to specific preparative procedures previous to examination.

The historical field of expertise of morphologists presents some degree of overlap with, on the one hand, that of clinical tissue imagers, and, on the other hand, that of cell and molecular biologists. However, for a long period of time, the technologies used by the various categories of cell and tissue imagers have evolved quite independently. The situation is now changing. New technologies progressively bridge the gaps still existing between the various levels of cell and tissue imaging. Other technological advances suggest that, in the near future, it would be possible to perform detailed morphological observations, not only ex vivo, as can be done today, but also in vivo.

The aims of this review are to describe the recent major technological advances in morphological imaging, to evaluate their potential for applications in related fields of imaging such as endoscopy, to investigate their consequences on the relations betweeen the various categories of cell and tissue imagers and to discuss the new challenges that must be faced by the pathologist.

\section{THE ROLE OF MORPHOLOGICAL TECHNIQUES IN CELL AND TISSUE IMAGING}

Morphological investigations may fulfill several roles. An important one is the structural analysis of cells and tissues. The description of the various types of cells and tissues, the analysis of their architecture and the delineation of criteria allowing for the correct identification of the various cell lineages have provided the basis necessary for the historical development of histology, embryology, and pathology. Today, structural criteria remain essential for the correct identification of cells and tissues in the normal state, for the monitoring of their changes during embryogenesis and organogenesis, and for the evaluation of their changes in disease. Historically, the structural analysis of cells and tissues has been based on descriptive morphological techniques, including conventional histology, histochemistry, and electron microscopy. Today, it may also rely on more recently developed techniques, such as immunohistochemistry, which permits the use of cell specific proteins as markers for the identification of cell lineages.

A second aim of morphological imaging is to contribute to the functional analysis of cells and 
tissues. Morphological techniques provide essential informations about the relations between structure and function in cells and tissues and, in this way, have largely contributed to the development of cell biology. In collaboration with other techniques, morphological techniques may also contribute to the understanding of metabolic and physiological processes in health and disease. The need for an accurate functional analysis of cells and tissues has had important consequences on the practice of morphological sciences: it prompted the development of new techniques allowing for in situ analysis of gene expression, such as immunohistochemistry and in situ hybridisation, as well as the development of new microscopical devices, such as confocal microscopy.

More recently, morphological techniques have been used to analyse in situ the genetic characteristics of normal or pathological cells. This resulted in the development of specific tools derived from molecular biology and molecular cytogenetics, such as fluorescent in situ hybridisation (FISH), and in the emergence of new preparative techniques, such as tissue microdissection.

\section{NEW INVESTIGATIVE TOOLS}

Several potent investigative tools have been developed in the past two decades by the adaptation to cellular and tissular preparations of techniques initially used in cell biology, molecular biology, or cytogenetics. These new techniques have made it possible to studying the in situ expression of specific proteins or of nucleic acids at the tissular, cellular, or subcellular levels. They include: immunohistochemistry, in situ hybridisation, in situ PCR, and in situ molecular cytogenetics (FISH and CISH).

\section{Immunohistochemistry}

Immunohistochemistry is not a new technique: it was developed around 1980 and gained a large acceptance by the 1990s. ${ }^{1}$ However, immunohistochemistry remains one of the most powerful investigative tools available to morphologists and has been the foundation on which most of the new morphological techniques developed. ${ }^{2}$ It therefore still deserves a special mention.

The aim of immunohistochemistry is to detect the presence of a specific protein (or peptide) within a cellular or tissular preparation. All the immunohistochemical techniques are based on the same basic principle: (a) a specific antibody raised to the protein of interest is allowed to react with the cellular or tissular preparation; (b) then, the antibody-antigen complex formed is revealed by a method permitting its detection by microscopy. Numerous variants of immunohistochemical techniques have been described. Initial efforts were mainly devoted to the standardisation of the techniques, the improvement of their specificity, and their adaptation to the various types of microscopical techniques, including conventional light microscopy, fluorescence microscopy, and electron microscopy.

An important breakthrough resulted from the development of the technology of monoclonal antibodies. So far, the antibodies used in immunohistochemistry were prepared by immunising laboratory animals with highly purified proteins. The technology of monoclonal antibodies made it possible to produce in vitro large amounts of antibodies of constant specificity and affinity. Moreover, and this was probably the most important consequence of this technological revolution, the advent of monoclonal antibodies helped to design new strategies to purify and identify proteins present in low amounts in complex biological structures. In turn, the characterisation of such proteins rapidly provided new tools for the identification of cell lineages and the understanding of their functions.

In recent years, great efforts have been made to improve the sensitivity of the immunohistochemical techniques. Amplification procedures have been designed to increase the contrast between the signal and the background. To permit the use of immunohistochemistry in a large variety of histological preparations, including fixed tissues, appropriate monoclonal antibodies have been selected and various techniques of antigen retrieval, including the controlled digestion of tissue sections by proteases or their microwave pretreatment, have been designed. Finally, techniques of whole tissue immunohistochemistry have been developed to permit the analysis of the distribution of a given protein at the level of a whole organ or, even, a whole organism, such as an embryo.

Applications of immunohistochemistry are numerous. In cell biology, immunohistochemistry remains a potent investigative tool used for structural and functional imaging. ${ }^{2}$ In pathology, immunohistochemistry is routinely used in diagnosis, prognostic evaluation and, more recently, pre-therapeutic assessment. ${ }^{3}$ The detection of specific proteins by labelled antibodies has been mainly restricted to tissue imaging "ex vivo" - that is, in cellular and tissular preparations. However, several studies show that labelled antibodies can also be used for in vivo functional imaging..$^{56}$

\section{In situ hybridisation and in situ PCR}

In situ hybridisation and in situ PCR aim to detect the presence of specific nucleic acid sequences within cellular or tissular preparations. ${ }^{78}$ They can be used to detect either DNA or RNA sequences. The principle of in situ hybridisation techniques is simple: (a) a molecular probe designed to interact with a specific nucleic acid sequence is allowed to react with a cellular and tissular preparation; (b) the hybrid formed in situ between the probe and its target is revealed by a method permitting its detection by light or electron microscopy.

The combination of PCR and in situ hybridisation has resulted in the development of in situ $\mathrm{PCR}^{9}{ }^{10}$; in this technique, a PCR technique is applied to cellular or tissular preparations to amplify the number of target sequences; amplicons are revealed either directly by incorporation of labeled nucleotides or by further in situ hybridisation with labelled probes. ${ }^{11}$

In situ hybridisation techniques provide informations complementary to those provided by immunohistochemistry. They make it possible to detect the nucleic acid sequences coding for the expression of specific proteins. The combination of immunohistochemistry and in situ hybridisation therefore allows for a complete and detailed analysis of gene expression in situ. ${ }^{12}$ However, in situ hybridisation techniques have proved to be difficult to standardise and to apply to routinely prepared samples. ${ }^{13}$ This largely explains why, in contrast with immunohistochemistry, in situ hybridisation and in situ PCR have not yet found routine applications in pathology, except for the detection of viral nucleic acid sequences. ${ }^{14}{ }^{15}$

\section{In situ molecular cytogenetics (FISH and $\mathrm{CISH}$ )}

In situ molecular cytogenetics aims to detect chromosomal sequences by using specific molecular probes able to hybridise with target regions of genomic DNA. ${ }^{16}$ The hybrid is then revealed by a method permitting its detection by a light microscope. The techniques using fluorescent labels, known as FISH, are the most commonly used. ${ }^{17}$ More recently, techniques using non-fluorescent labels (CISH) and working on conventionnally prepared tissues, have been described.

In situ molecular cytogenetic techniques were first developed for use on chromosomal preparations but have soon been adapted to cellular or tissular preparations. ${ }^{18}$ Applications of molecular cytogenetics in situ include the identification of specific chromosomes (such as the detection of chimerism in transplant organs through the identification of sex chromosomes), the detection of numerical chromosomic alterations (such as the loss or the amplification of specific chromosomes in tumours) and the caryotyping of tumour cells.

So far, FISH (or CISH) have been mainly applied to cell and tissue samples for research purposes. However, it is probable 
that this technique may soon find applications in routine pathology. A first example is the detection of the amplification of the gene coding for the Her2/neu protein in breast cancer. ${ }^{19}$ Patients with breast cancers overexpressing the Her2/neu protein, as a result of the amplification of the corresponding gene located on chromosome 17, are eligible for a specific therapy based on the administration of humanised anti-Her $2 / n e u$ antibodies. So far, the identification of such patients was routinely based on the immunohistochemical evaluation of the expression level of Her2/neu by tumour cells. However, it has recently been suggested that the direct demonstration of the amplification of the corresponding gene by FISH may be more sensitive than conventional immunohistochemistry for the identification of patients eligible for specific anti-Her2/neu therapy. It is probable that other targets would be rapidly identified for such diagnostic procedures. A good candidate in gastrointestinal pathology is the EGF receptor gene, amplified in a significant proportion of digestive cancers and to which specific inhibitors have been recently raised.

\section{NEW MICROSCOPIC TOOLS Confocal microscopy}

Confocal microscopy was developed in the 1980s. It combines a new technological approach allowing for the optical sectioning of thick cellular or tissular samples with the use of fluorescent markers able to identify specific cellular targets. ${ }^{2021}$

In conventional microscopy, informations come not only from the focal plane of observation of the object, but also from the planes located below and above from this plane; this makes it impossible to examine thick objects as a whole and requires previous physical sectioning to obtain thin sections minimising out of focus informations. In contrast, confocal microscopy uses informations coming only from the focal plane of the object and rejects the out of focus informations coming from the points located above and below the focal plane. Confocal microscopy makes it therefore possible to examine thick biological objects, such as whole cells or tissue sections of up to $150 \mu \mathrm{m}$, without previous physical sectioning. In addition, confocal microscopy is able to achieve better resolutions than conventional microscopy in most situations, and therefore, to provide better and more detailed images than can be obtained with standard microscopic examination.

Confocal microscopy is based on the illumination of the biological specimen by an excitation light followed by the detection of the emitted light. This implies that only fluorescent objects can be examined. In most cases, the biological specimen is made fluorescent by the use of labels directed to specific intracellular or intratissular structures (confocal epifluorescence). More recently, the spontaneous autofluorescence of many biological molecules (confocal epitransmission) or the capacity of opaque surfaces to reflect light (confocal reflectance) have been exploited.

The rejection of out of focus interference, which is the main characteristic of confocal microscopy, is achieved by two ways: (a) the illumination of the specimen by the excitation light is restricted to a single point, usually by focusing one (or more) parallel laser beam in the focal plane of the specimen; the illumination intensity therefore drops off sharply above and below the focal plane; in contrast, in conventional microscopy, the excitation light illuminates uniformly the whole specimen; (b) the detection of the emitted light is made through a pinhole aperture located in a conjugated focal plane to the specimen to block any light coming away from the point currently illuminated; in contrast, in conventional microscopy, the emitted light passes through a chromatic reflector directly to the eyepiece.

In confocal microscopy, the examination of the specimen is achieved by a method of optical sectioning: the scanning of the specimen by the illumination light is made through successive sections across the whole thickness of the specimen. A computer system is used to control the correct sequential scanning of the specimen, to assemble the image for display onto a video monitor and to provide, if necessary, a three dimensional reconstruction of the distribution of the fluorescent label within the whole specimen.

The main advantages of confocal microscopy over conventional microscopy are: (a) from the "optical" point of view, the higher resolution of the images provided and the capacity to obtain very precise tridimensional informations; (b) from the "biological" point of view, the capacity to examine whole specimens with their original structure preserved, in the absence of the artefacts resulting from the previous physical sectioning required in conventional microscopy. Confocal microscopy is largely used in cell biology and provides very important informations for the understanding of the relations between structure and function in biological specimens. However, this technique has not yet found its way in routine pathology. This may be explained by its constraints (expensive equipment, necessity to use fluorescent labels). Moreover, it remains to be demonstrated that the informations provided by confocal microscopy may be more useful than those provided by conventional microscopy for routine diagnosis.

\section{Multiphoton microscopy}

In some situations, such as the examination of very thick specimens or the analysis of living biological specimens for long periods of time, confocal microscopy is not well adapted. This has led to the development of a new technique, known as multiphoton microscopy. ${ }^{22}$

Multiphoton microscopy is built up on an optical principle known since 1931 but unexploited until recently. Like confocal microscopy, multiphoton microscopy is based on the excitation of fluorescent molecules (either intrinsic or extrinsic to the tissue examined) by photons emitted by a laser. However, instead of using a single photon of ultraviolet light as the source of excitation, multiphoton microscopy uses two photons (or more) of red/infrared light absorbed simultaneously by the fluorescent molecule. This is achieved by using ultrafast near infrared lasers. The main advantage of the technique is that the red light used for illuminating the specimen cannot cause fluoresence emission in another plane other than the focal plane, where the red/infrared light is at its peak. This is a decisive advantage as compared with conventional confocal microscopy, in which the fluorescence emission is most intense at the focal plane but may also come from throughout the beam path. Therefore, in multiphoton microscopy, there is no need for a confocal pinhole to reduce the out of focus emitted light.

The main advantages of multiphoton microscopy as compared with conventional confocal microscopy may be summarised as follows: (a) red or infrared light is less scattering and penetrates deeper in tissues; this makes therefore it possible to examining thicker specimens than can be done with conventional confocal microscopy; (b) the red/infrared light used in multiphoton microscopy is less toxic to living specimens than the ultraviolet light used in conventional confocal microscopy, which makes it possible to examining living specimens for longer periods of time; (c) multiphoton excitation provides highly localised spatial control of fluoresence excitation and emission, which would be essential for certain emerging applications of in vivo optical imaging and for optical micromanipulation. ${ }^{24} 25$

Multiphoton microscopy promises to be a very potent tool for the imaging of thick tissues, both ex vivo and in vivo, and of the metabolic activity of living specimens. ${ }^{26}$ However, some of its current limitations must be underlined: (a) the resolution is slightly lower than in conventional confocal microscopy; (b) some damage may occur in tissues containing chromophores able to absorb the red/infrared light, such as melanin; (c) the equipements are expensive and their use requires a high degree of expertise. 


\section{Atomic force microscopy and scanning probe} microscopy

We would only cite here the existence of new microscopic tools aimed to imaging single molecules or macromolecules. The two most important ones are atomic force microscopy and scanning probe microscopy. So far, only research applications have been described but, in the future, these new techniques may prove to be adapted for surface imaging of complex biological structures..$^{27-29}$

\section{NEW PREPARATIVE TOOLS}

In recent years, new techniques have been developed to improve the preparation of tissue specimens to fulfill the requirements of molecular biology techniques and of proteomic analysis.

\section{Tissue microdissection}

The aims of tissue microdissection are to select and pick up single cells or groups of cells from a heterogeneous tissue sample to perform molecular analyses. The emergence of tissue microdissection is an indirect consequence of the large development of PCR techniques, which have been made it possible to perform molecular analyses from very limited amounts of biological material, but which require very pure preparations to avoid any risk of contamination. ${ }^{30}$

Microdissection techniques are used as a preparative step in the analysis of heterogeneous tissues or complex lesions, containing numerous cell types or numerous cell lineages. ${ }^{31}{ }^{32}$ For instance, a tumour contains not only tumour cells, but also stromal cells (fibroblasts and endothelial cells) and inflammatory cells; in some tumours, the number of tumour cells may actually be much lower than that of non-tumour cells. Conventional preparative techniques for molecular analyses, based on whole tissue dissociation and homogeneisation, are non-selective and do not take into account the heterogeneity of the initial sample. This may result in a decrease in the specificity and sensitivity of the molecular techniques and to biases in the interpretation of the results. To avoid such difficulties, a preparative step permitting the enrichment of the preparation in selected cell populations must be performed. Tissue microdissection is one of the methods available for this purpose. Its advantages are: (a) a higher sensitivity than other preparative techniques, such as cell sorting, (b) the possibility to perform concomittant topographical analyses.

Tissue microdissection techniques comprise three main steps: (a) the selection of the cells of interest, usually achieved under microscopical control, and which may be made easier by the use of cell markers previously revealed by immunohistochemical or in situ hybridisation techniques, (b) the dissection of the cells of interest, (c) the collection of the dissected material, which must be closely controlled to avoid contaminations.

Three main techniques of tissue microdissection may be used: manual techniques, micromanipulator assisted techniques, and laser assisted techniques. Manual techniques are the simplest ones. After selection under microscopical control, the cells of interest are picked up by using a sharp pipette guided by hand. The use of a micromanipulator to guide the movement of the hand results in a considerable gain in precision, at the expense of the output. Laser assisted techniques, developed only recently, have rapidly gained a large acceptance: they combine precision, high output, and close control of material collection. Several variants of laser assisted techniques have been developed. The best known are laser capture and laser microdissection (or pressure). In laser capture, the histological preparation is covered with a thermosensitive plastic film; impacts of predefined diameter are then performed by using an infrared laser positioned above the preparation; this allows the thermosensitive film to adhere to the histological preparation; the zone of impact is then cut off and directly immmerged in extraction buffer. ${ }^{33}$ In laser microdissection (or pressure), ${ }^{34}$ cutting ultraviolet lasers positioned either above or below the histological preparation are used to precisely cut off the zone(s) of interest within the tissue sample; according to the system, the dissected material is then either catapulted within the extraction buffer by a specifically designed device or, more simply, collected by gravity within a tube filled by extraction buffer. Several manufacturers have now developed various systems based on variants of either laser capture or laser microdissection.

Laser assisted tissue microdissection techniques have many advantages over the other techniques of microdissection. They are adapted to most protocols, including those requiring a great precision in the dissection, such as the picking up of one cell, as well as those requiring the dissection of a large amount of material. Another advantage is the good control of the collection of the dissected material, which avoids most contaminations. However, manual techniques retain their indications: simple and unexpensive, they permit the rapid obtention of large amounts of material. They remain the first choice when the level of precision required is low and the amount of material required is important.

So far, tissue microdissection has found its main applications in tumour pathology. Tissue microdissection has been used: (a) to enrich the material submitted to molecular analysis in tumour cells, by selectively picking them up and eliminating stromal cells, (b) to compare different zones or different cell populations within the same tumour, (c) to compare the molecular profile of tumour cells and adjacent normal periturmoral cells, (d) to compare the molecular profile of neoplastic cells isolated from areas of dysplasia, in situ carcinoma, and invasive carcinoma coexisting within the same tissue sample.

So far, the material obtained by tissue microdissection has mainly been used for DNA analysis (loss of heterozygosity, microsatellite instability, mutation analysis). ${ }^{35-37}$ Some protocols may be applied in integrated diagnostic strategies. A good example is the demonstration of microsatellite instability for the diagnosis of HNPCC syndromes or the identification of MSI positive colon cancers. The same tissue samples may be used for the immunohistochemical screening of mismatch repair enzyme expression, then, after microdissection of normal colonic mucosa and tumour tissue respectively, for the demonstration of microsatellite instability by PCR and finally, for mutation analysis.

More recently, material obtained by tissue microdissection of frozen samples has proved to be adapted for the study of the transcriptome, ${ }^{38}$ including by the most recent techniques such as quantitative real time PCR or biochips, and for the sudy of the proteome, including by high input analytical techniques such as the so called protein chips..$^{39}$

\section{Tissue arrays}

Low input and lack of reproducibility are among the major criticisms usually made to morphological techniques. Tissue arrays may help to overcome these limitations.

The aim of tissue arrays is to permit the simultaneous analysis by immunohistochemistry or in situ hybridisation of a large number of tissue preparations, each representative of a different initial tissue sample. ${ }^{41-43}$ To achieve this purpose, a comparatively simple device has been designed to drill cylinders of variable diameters within the initial blocks of tissue and arrange them, side by side, to form a new block. Sections are then cut across the new block according to standard histological techniques. Slides are prepared and may be submitted to conventional immunohistochemical or in situ hybridisation techniques without specific adaptation.

Tissue arrayed blocks may contain up to 1000 specimens, but the actual number in routine practice is usually limited from 300 to 600. A degree of technical expertise is required for the preparation of the arrayed block and its sectioning. 
Reproducibility and high input are the main interests of the technique of tissue arrays. ${ }^{44}$ An important question is to determine to what extent the specimens taken off the initial blocks are actually representative of the initial sample. This is particularly important as tumour cells from different areas of a given lesion may present significant differences in their phenotypic and genotypic characteristics. This issue has been investigated by several studies, which concur to show that the concordance between tissue arrays and the initial samples is usually greater than $90 \%$.

\section{CELL AND TISSUE IMAGING IN SITU: BRIDGING THE GAPS}

Recent technological advances suggest that the gaps still observed between the various techniques of tissue imaging, and the various categories of tissue imagers, will be progressively reduced in the near future. On the one hand, new techniques of tissue imaging are able to achieve resolutions close to those obtained to conventional histological techniques. On the other hand, techniques initially developed for ex vivo cell imaging are now adapted to perform in vivo histology or intravital microscopy.

\section{Scaling down tissue imaging: the advent of micro-radiology and of high resolution endoscopy}

The development of micro-radiology - that is, miniaturised radiological methods of tissue imaging, has been prompted by the emergence of animal models of human diseases. ${ }^{45}$ The degree of resolution of some micro-radiological techniques is now comparable to that of conventional morphological techniques. Micro-RMN techniques may achieve resolutions of $10 \mu \mathrm{m}$. Micro-computed tomography, or high resolution $x$ ray computed tomography, is able to produce highly detailed three dimensional images of soft tissues or bone structures, including the rendering of structures as small as $20 \mu \mathrm{m}$ in diameter. Direct comparisons with histological preparations may be performed. Ultrasound biomicroscopy, based on the same principle than conventional diagnostic ultrasound examinations, uses high operating frequencies able to achieve resolutions ranging from $30 \mu \mathrm{m}$ to $100 \mu \mathrm{m}$. Ultrasound biomicroscopy permits real time imaging of physiological events and detailed analysis of tissue organogenesis. So far, these techniques are applied to the mouse. However, it is probable that, in the near future, these technological advances will be adapted to clinical radiology and may improve the quality of tissue imaging in patients.

In the same way, technological advances have allowed endoscopy to achieve high levels of resolution in the rendering of the architecture of digestive mucosa. ${ }^{46}{ }^{47}$ Several of these new techniques, such as high resolution endoscopy, chromoscopy, fluorescence endoscopy, light scattering reflectance, spectroscopy, and optical coherence tomography, will be presented in detail elsewhere during this symposium. Resolutions as low as $10 \mu \mathrm{m}$ may be achieved by some of these techniques, such as optical coherence tomography. ${ }^{48}$

\section{Scaling up microscopical imaging: the advent of in vivo histology and intravital microscopy}

Confocal microscopy and multiphoton microscopy, initially developed for ex vivo imaging of cells and tissues, have been recently applied to in vivo imaging, through the adaptation of specific optical devices, such as in tandem scanning confocal microscopy. These new technologies have been successfully used for the in vivo examination of living tissues, ${ }^{49}{ }^{50}$ such as the liver, ${ }^{21}$ the kidney, ${ }^{51}$ and the colon, and for real time in vivo study of physiological or physiopathological processes, such as drug related injury of the colon, acute inflammation, wound healing, ${ }^{5253}$ and angiogenesis. ${ }^{5455}$

It has recently been proposed to combine endoscopy with scanning confocal microscopy for diagnostic purposes, either to build endomicroscopes ${ }^{56}$ or to examine fresh endoscopic biopsy fragments through a confocal microscope, to achieve "extemporaneous" diagnosis of gastrointestinal tract lesions. ${ }^{47}$ This is not, strictly speaking, in vivo histology, but nevertheless challenges the use of conventional morphological techniques to achieve the correct diagnosis of a lesion.

\section{CELL AND TISSUE IMAGING IN SITU: NEW CHALLENGES FOR THE PATHOLOGIST}

With the advent of new technologies, pathologists must be prepared to face new challenges. We will briefly discuss some of them.

\section{Progress in endoscopy and radiology: Which role for} the histological examination in diagnosis?

Histopathological examination is currently considered as the gold standard for the diagnosis of neoplastic lesions of the gastrointestinal tract as well as for their grading and staging. Some authors suggest that this may be challenged in the future as a consequence of the development of new techniques of clinical imaging. There are already some examples of lesions for which a histopathological diagnosis is no more longer considered necessary, because of their characteristic appearance at imaging studies; for instance, the diagnosis of typical cases of focal nodular hyperplasia of the liver or serous cystadenomas of the pancreas can be confidently made at imaging studies and does not require biopsy. Currently, efforts are made to increase the performances of the endoscopical diagnosis of the neoplastic lesions of the gastrointestinal tract. For instance, the use of high resolution endoscopy, combined with chromoscopy, may be used in the the differential diagnosis between neoplastic and non-neoplastic lesions (such as the distinction between adenomas and hyperplastic polyps in the colon) or the grading of mucosal neoplastic lesions ( such as the grading of colic adenomas or that of intraepithelial neoplasia in Barrett's mucosa). Current endoscopical classifications are based on the so called pit patterns-that is, on the architectural appearance of the polypoid (and even nonpolypoid) lesions of the gastrointestinal tract. ${ }^{57}$ It must however be recalled that the histological grading of the neoplastic lesions of the gastrointestinal tract is based on the degree of cellular and nuclear atypia, which may not be correlated with the degree of architectural remodelling. Another emerging technique, light scattered reflectance, gives informations about the size of nuclei and their density in a given area and therefore uses the same criteria than the histopathological examination. ${ }^{58}$ Assuming that all these techniques could be successfully adapted to the clinical practice and used during routine examinations, their first application will probably be to direct endoscopic biopsies by a better identification of the areas of the most important diagnostic relevance. Will such techniques actually avoid the need for diagnostic biopsies in the future? It is too early to answer this question but a few points may be made: (a) the histological examination of biopsy specimens will probably remain for a long time as a simple way to obtain a reliable diagnosis in many non-specialised centres, (b) tissue sampling may remain necessary for purposes other than diagnosis, such as prognostic evaluation and pre-therapeutic assessment.

\section{Progress in molecular biology: Which role for the} pathologist in tumour biology?

Currently, the pathological examination of tissue specimens plays an important part in prognostic evaluation and pretherapeutic assessment. This role is challenged by the emergence of new and powerful techniques derived from molecular biology. The molecular profiling of tumour specimens by techniques such as DNA arrays has already shown its capacity to identify subgroups of prognostic and therapeutic relevance within subsets of morphologically homogeneous tumours. In 
the near future, such techniques will probably become essential for the accurate treatment of the patients. However, their actual field of application remains to be determined. In any case, pathologists will retain important roles. They will be directly involved in the collection and storage of the tissues necessary to perform molecular analyses, and their morphological expertise will be necessary to provide molecular biologists with the right material to examine. Moreover, it cannot be excluded that, after an initial phase in which molecular techniques will be necessary to identify the genetic alterations of clinical relevance, it will be possible to develop simple diagnostic tools based on conventional techniques, such as immunohistochemistry. Oncohaematology provides several examples of this kind.

\section{CONCLUSION}

Numerous new techniques of cell and tissue imaging have been developed during the past decades. On the one hand, some of them, such as FISH ${ }^{59}$ or tissue microdissection, enable pathologists to further extend the informations that they may obtain from tissue and cell analysis. However, on the other hand, other emerging techniques may challenge the traditional roles of pathologists in diagnosis and prognostic evaluation. Technological changes may therefore lead in the future to the redifinition of the historical field of expertise of pathology but will surely open the way to new modes of communication between cell and tissue imagers.

\section{REFERENCES}

1 Swanson PE. Foundations of immunohistochemistry. A practical review. Am J Clin Pathol 1988;90:333-9.

2 Johnson CW. Issues in immunohistochemistry. Toxicol Pathol 1999;27:246-8

3 Carbone A. Will the "new biology" affect the future of histopathology? Int J Biol Markers 2001; 16:1-4.

4 Bodey B. The significance of immunohistochemistry in the diagnosis and therapy of neoplasms. Expert Opin Biol Ther 2002;2:371-93.

5 Von Kleist S. Ten years of tumor imaging with labelled antibodies. In Vivo 1993:7:581-4

6 Sipkins DA, Cheresh DA, Kazemi MR, et al. Detection of tumor angiogenesis in vivo by alphaVbeta3-targeted magnetic resonance imaging. Nat Med 1998:4:623-6.

7 Mitchell BS, Dhami D, Schumacher U. In situ hybridisation: a review of methodologies and applications in the biomedical sciences. Med Lab Sci 1992;49:107-18.

8 DeLellis RA. In situ hybridization techniques for the analysis of gene expression: applications in tumor pathology. Hum Pathol 1994;25:580-5

9 Nuovo GJ. PCR in situ hybridization. Methods Mol Biol 1994;33:223-41.

10 Nuovo GJ. In situ PCR: protocols and applications. PCR Methods Appl 1995:4:S151-67.

11 Nuovo GJ. In situ localization of PCR-amplified DNA and cDNA. Mol Biotechnol 1998; 10:49-62.

12 Nuovo GJ. Co-labeling using in situ PCR: a review. J Histochem Cytochem 2001:49:1329-39.

13 Stoler MH. In situ hybridization. A research technique or routine diagnostic test? Arch Pathol Lab Med 1993;117:478-81.

14 Nuovo GJ. Detection of viral infections by in situ PCR: theoretical considerations and possible value in diagnostic pathology. J Clin Lab Anal 1996:10:335-49.

15 McNicol AM, Farquharson MA. In situ hybridization and its diagnostic applications in pathology. J Pathol 1997;182:250-61

16 Tibiletti MG, Bernasconi B, Dionigi A, et al. The applications of FISH in tumor pathology. Adv Clin Pathol 1999;3:111-18.

17 Gozzetti A, Le Beau MM. Fluorescence in situ hybridization: uses and limitations. Semin Hematol 2000;37:320-33.

18 Ried T, Schrock E, Ning Y, et al. Chromosome painting: a useful art. Hum Mol Genet 1998;7:1619-26.

19 Ross JS, Fletcher JA. HER-2/neu (c-erb-B2) gene and protein in breast cancer. Am J Clin Pathol 1999:112:S53-67.

20 Imbert D, Hoogstraate J, Marttin E, et al. Imaging thick tissues with confocal microscopy. Methods Mol Biol 1999;122:341-55.

21 Tadrous PJ. Methods for imaging the structure and function of living tissues and cells: 3 . Confocal microscopy and micro-radiology. J Pathol 2000; 191:345-54.

22 Denk W, Strickler JH, Webb WW. Two-photon laser scanning fluorescence microscopy. Science 1990;248:73-6.

23 Diaspro A. Introduction to two-photon microscopy. Microsc Res Tech 1999:47: 163-4

24 Mitchison TJ, Sawin KE, Theriot JA, et al. Caged fluorescent probes. Methods Enzymol 1998;291:63-78.
25 Tadrous PJ. Methods for imaging the structure and function of living tissues and cells: 2 . Fluorescence lifetime imaging. J Pathol 2000;191:229-34

26 Piston DW, Knobel SM. Quantitative imaging of metabolism by two-photon excitation microscopy. Methods Enzymol 1999;307:351-68.

27 Czajkowsky DM, Iwamoto H, Shao Z. Atomic force microscopy in structural biology: from the subcellular to the submolecular. J Electron Microsc (Tokyo) 2000;49:395-406.

28 Hinterdorfer $\mathbf{P}$, Schutz $G$, Kienberger $F$, et al. Detection and characterization of single biomolecules at surfaces. J Biotechnol 2001:82:25-35.

29 Fotiadis D, Scheuring S, Muller SA, et al. Imaging and manipulation of biological structures with the AFM. Micron 2002;33:385-97.

30 Going JJ, Lamb RF. Practical histological microdissection for PCR analysis. J Pathol 1996;179:121-4

31 Sirivatanauksorn Y, Drury R, Crnogorac-Jurcevic T, et al. Laser-assisted microdissection: applications in molecular pathology. J Pathol $1999 \cdot 189 \cdot 150-4$

32 Gillespie JW, Ahram M, Best CJ, et al. The role of tissue microdissection in cancer research. Cancer J 2001;7:32-9.

33 Bonner RF, Emmert-Buck M, Cole K, et al. Laser capture microdissection: molecular analysis of tissue. Science 1997:278:1481-3.

34 Bohm M, Wieland I, Schutze K, et al. Microbeam MOMeNT non-contact laser microdissection of membrane-mounted native tissue. Am J Pathol 1997;151:63-7.

35 Walch A, Komminoth P, Hutzler P, et al. Microdissection of tissue sections: application to the molecular genetic characterisation of premalignant lesions. Pathobiology 2000;68:9-17.

36 Walch A, Specht K, Smida J, et al. Tissue microdissection techniques in quantitative genome and gene expression analyses. Histochem Cell Biol 2001:115:269-76.

37 Best CJ, Emmert-Buck MR. Molecular profiling of tissue samples using laser capture microdissection. Expert Rev Mol Diagn 2001;1:53-60.

38 Specht K, Richter T, Muller U, et al. Quantitative gene expression analysis in microdissected archival formalin-fixed and paraffin-embedded tumor tissue. Am J Pathol 2001;158:419-29.

39 von Eggeling F, Davies H, Lomas L, et al. Tissue-specific microdissection coupled with ProteinChip array technologies: applications in cancer research. Biotechniques 2000;29:1066-70

40 Craven RA, Banks RE. Laser capture microdissection and proteomics: possibilities and limitation. Proteomics 2001;1:1200-4.

41 Bubendorf L, Nocito A, Moch H, et al. Tissue microarray (TMA) technology: miniaturized pathology archives for high-throughput in situ studies. J Pathol 2001;195:72-9.

42 Horvath L, Henshall S. The application of tissue microarrays to cancer research. Pathology 2001;33:125-9.

43 Kallioniemi OP, Wagner U, Kononen J, et al. Tissue microarray technology for high-throughput molecular profiling of cancer. Hum Mol Genet 2001; 10:657-62

44 Moch $\mathbf{H}$, Kononen T, Kallioniemi OP, et al. Tissue microarrays: what will they bring to molecular and anatomic pathology? Adv Anat Pathol 2001;8:14-20.

45 Weissleder R. Scaling down imaging: molecular mapping of cancer in mice. Nat Rev 2001;2:1-8.

46 Mallery S, Van Dam J. Endoscopic practice at the start of the new millennium. Gastroenterology 2000;1 18:S129-47.

47 Mallery S, Van Dam J. Advances in diagnostic and therapeutic endoscopy. Med Clin North Am 2000;84:1059-83.

48 Tadrous PJ. Methods for imaging the structure and function of living tissues and cells: 1. Optical coherence tomography. J Pathol 2000:191:115-19.

49 Petran M, Hadravsky M. Tandem scanning microscope-a new tool for three-dimensional microanatomy. Prog Clin Biol Res 1989;295:551-8.

50 Jester JV, Andrews PM, Petroll WM, et al. In vivo, real-time confocal imaging. J Electron Microsc Tech 1991;18:50-60.

51 Andrews PM, Petroll WM, Cavanagh HD, et al. Tandem scanning confocal microscopy (TSCM) of normal and ischemic living kidneys. Am J Anat 1991;191:95-102.

52 Chew SJ, Beuerman RW, Kaufman HE. Real-time confocal microscopy of keratocyte activity in wound healing after cryoablation in rabbit corneas. Scanning 1994:16:269-74.

53 Chew SJ, Beuerman RW, Kaufman HE, et al. In vivo confocal microscopy of corneal wound healing after excimer laser photorefractive keratectomy. Clao J 1995;21:273-80.

54 Merchant FA, Aggarwal SJ, Diller KR, et al. In-vivo analysis of angiogenesis and revascularization of transplanted pancreatic islets using confocal microscopy. J Microsc 1994;176:262-75

55 Merchant FA, Diller KR, Aggarwal SJ, et al. Angiogenesis in cultured and cryopreserved pancreatic islet grafts. Transplantation 1997;63:1652-60.

56 Delaney PM, King RG, Lambert JR, et al. Fibre optic confocal imaging (FOCI) for subsurface microscopy of the colon in vivo. J Anat 1994; 184:157-60.

57 Tung SY, Wu CS, Su MY. Magnifying colonoscopy in differentiating neoplastic from nonneoplastic colorectal lesions. Am J Gastroenterol 2001;96:2628-32

58 Georgakoudi I, Jacobson BC, Van Dam J, et al. Fluorescence, reflectance, and light-scattering spectroscopy for evaluating dysplasia in patients with Barrett's esophagus. Gastroenterology 2001;120:1620-9.

59 Geboes K. Barrett's esophagus: the metaplasia-dysplasia-carcinoma sequence: morphological aspects. Acta Gastroenterol Belg 2000;63:13-17. 